\title{
Aging impairs insulin-stimulated glucose uptake in rat skeletal muscle via suppressing AMPK $\alpha$
}

\author{
Wan Qiang ${ }^{1 *}$, Kang Weiqiang ${ }^{2 *}$, \\ Zhu Qing ${ }^{3}$, Zhang Pengju ${ }^{4}$ \\ and Liu $\mathrm{Yi}^{1,5}$ \\ ${ }^{1}$ Department of Internal Medicine \\ Shandong Provincial Hospital Shandong University \\ Jinan 250021, China \\ ${ }^{2}$ Department of Internal Medicine \\ Qingdao Municipal Hospital \\ Qingdao 266011, China \\ ${ }^{3}$ Department of Internal Medicine \\ Qingdao Haici Hospital \\ Qingdao 266033, China \\ ${ }^{4}$ Department of Biochemistry \\ Shandong University \\ Jinan 250012, China \\ ${ }^{5}$ Corresponding author: Tel, 86-053-185758915; \\ Fax, 86-053-186616046; Email, liuyishanyi@yahoo.com.cn \\ ${ }^{*}$ These authors contributed equally to this work.
}

Accepted 20 June 2007

Abbreviations: ACC, acetyl-CoA carboxylase; AICAR, 5-aminoimidazole-4-carboxamide riboside; AMPK, AMP-activated protein kinase; CPT1, carnitine palmitoyl transferase 1; GluT4, glucose transporter 4; TG, triglyceride

\begin{abstract}
Insufficient intracellular fat oxidation is an important contributor to aging-related insulin resistance, while the precise mechanism underlying is unclear. AMP-activated protein kinase (AMPK) is an important regulator of intracellular fat oxidation and was evidenced to play a key role in high-glucose and high-fat induced glucose intolerance. In the present study, we investigated whether altered AMPK expression or activity was also involved in aging-related insulin resistance. Insulin sensitivity of rats' skeletal muscles was evaluated using in-vitroglucose uptake assay. Activity of $\alpha$ subunit of AMPK (AMPK $\alpha$ ) was evaluated by measuring the phosphorylation of both AMPK $\alpha$ (P-AMPK $\alpha$ ) and acetyl-CoA carboxylase (P-ACC), while expression of AMPK $\alpha$ was assessed by determining the mRNA levels of AMPK $\alpha 1$ and AMPK $\alpha 2$, and protein contents of AMPK $\alpha$. Compared with 4-month old rats, 24-month old rats exhibited obviously impaired insulin
\end{abstract}

sensitivity. At the same time, AMPK $\alpha$ activity significantly decreased, while AMPK $\alpha$ expression did not alter during aging. Glucose transporter 4 expression also decreased in old rats. Compared with 24-month old rats, administration of the specific activator of AMPK, 5-aminoimidazole-4-carboxamide riboside (AICAR), significantly elevated AMPK $\alpha$ activity and GluT4 expression. Also, aging-related insulin resistance was significantly ameliorated by AICAR treatment. In conclusion, aging-related insulin resistance is associated with impaired AMPK $\alpha$ activity and could be ameliorated by AICAR, thus indicating a possible role of AMPK in aging-induced insulin resistance.

Keywords: 5'-AMP-activated protein kinase; aging; glucose transporter type 4; insulin resistance; muscle, skeletal; rat

\section{Introduction}

Aging is one of the single risk factor leading to insulin resistance and type 2 diabetes (Carvalho et al., 1996; Boirie et al., 2001; Scheen, 2005), while the precise mechanism is not fully elucidated yet. Recent researches have proposed that insufficient intracellular fatty acid oxidation and subnormal triglyceride (TG) accumulation in insulin target organs might be an important contributor (Cree et al., 2004; lossa et al., 2004; Menshikova et al., 2006; Slawik and Vidal-Puig, 2006). Although the effects of aging on impaired fatty acid oxidation and fat accumulation have been well demonstrated in both humans and rodents (CallesEscandon et al., 1995,1997; Park et al., 2006), the mechanism underlying is unclear. Part of the defect in fat oxidation might be explained by the altered body composition during aging, such as decreased muscle mass and increased fat mass (CallesEscandon et al., 1995). However, related studies showed differences of fat oxidation still exist when body composition was taken into account (Sial et al., 1996, 1998). Also, it was proposed that insufficient physical exercise in the eldly might play a role in aging-associated fat metabolism disturbance (Seals et al., 1984; Cox et al., 1999). But data from Short KR revealed that four months of physical training showed no obvious amelioration of the age-related insulin resistance (Short et al., 
2003). Taken all these data into consideration, we hypothesized there might be other mechanisms underlying aging-related fat oxidation insufficiency and insulin resistance.

In recent years, AMP-activated protein kinase (AMPK) has drawn a great deal of interest due to its regulative effect on intracellular fatty acid oxidation and insulin sensitivity. Several stresses such as exercise, hypoxia, or prolonged starvation could activate AMPK via increasing the ratio of intracellular AMP/ATP. Once activated, its classical effect is to inactivate acetyl-CoA carboxylase (ACC) in a phosphorylation manner, resulting in a decrease in malonyl-CoA, thus relieving inhibition of carnitine palmitoyltransferase 1 (CPT-1) and facilitating FFA entry into mitochondria for $\beta$-oxidation (Rutter et al., 2003). Via regulating intracellular fatty acid oxidation, AMPK has been proposed as a positive regulator of insulin sensitivity. Several physiological and pharmacological agents reportedly could exert their insulin-sensitizing effects via activating AMPK, such as leptin (Minokoshi et al., 2002), adiponection (Yamauchi et al., 2002) and metformin (Zhou et al., 2001). Besides regulating fatty acid oxidation, it was reported AMPK could regulate insulin sensitivity via stimulating Glut4 expression (Jessen et al., 2003). Further research demonstrated such the effect was mediated by MEF2 and GEF, two key Glut4 transcription regulators (Holmes et al., 2005).

Recently, Kraegen EW and colleagues reported that four days of glucose infusion induced a significant decrement of AMPK activities in rats' muscles and liver, accompanied with impaired insulin signaling and glucose disposal, indicating a possible role of AMPK in glucotoxicity (Kraegen et al., 2006). Our previous study also demonstrated that impaired expression and activity of AMPK, and accordingly insufficient intracellular fatty acid oxidation, might also participate in high-fat associated insulin resistance (lipotoxicity) (Liu et al., 2006). Whether such the mechanism is also involved in age-related insulin resistance is unclear. The present study investigated the role of AMPK in aginginduced insulin resistance and possible downstream mechanism underlying.

\section{Materials and Methods}

\section{Animals}

Total three groups of male Wistar rats (Shandong Epidemic-Prevention Station, Jinan, China) are used in the present study: young rats (4 month old, $n=9$ ); old rats (24 month old, $n=9$ ); old rats treated with 5-aminoimidazole-4-carboxamide riboside (Al-
CAR, Sigma, $n=9$ ); AICAR, the specific activator of AMPK, was administrated for a week via subcutaneous injection once a day at the dosage 0.5 $\mathrm{mg} / \mathrm{g} \mathrm{BW}$. Animals were housed in individual cages under conditions of constant temperature $\left(25^{\circ} \mathrm{C}\right)$ and humidity. Foods and water were provided adlibitum and maintained under 12L:12D lighting conditions (light on 6 am to $6 \mathrm{pm}$ ). All animal experimental procedures above were approved by Shandong University Institutional Animal Care and Use Committee.

\section{Biochemical analysis}

Blood samples were collected from inferior vena cava on the day rats were sacrificed. Fasting blood glucose levels were measured using glucose-oxidase method. Fasting serum insulin levels were determined by radioimmunoassay using an insulin reagent kit (Northern Bioengineering Institute, China). Levels of triglyceride (TG) and total cholesterol were detected using automatic biochemistry analyzer.

\section{In-vitro 2-deoxy glucose uptake}

In vitro glucose uptake was performed as previously described (Wan et al., 2005; Liu et al., 2006). Briefly, after rats were anesthetized (pentobarbital sodium, $50 \mathrm{mg} / \mathrm{kg} \mathrm{BW}$ ), gastrocnemius muscles were dissected out and rapidly cut into 40-80 $\mathrm{mg}$ strips. The muscle strips were put into $20 \mathrm{ml}$ flasks, containing $2.0 \mathrm{ml} \mathrm{KHB}$ buffer supplemented with $8 \mathrm{mM}$ glucose, $32 \mathrm{mM}$ mannitol, and $0.1 \%$ BSA and incubated at $35^{\circ} \mathrm{C}$ for $60 \mathrm{~min}$. Then, muscles were incubated for 20 min using KHB buffer supplemented with (for measurement of insulin-stimulated glucose uptake) or without (for measurement of basal glucose uptake) $2 \mathrm{mU} / \mathrm{ml}$ insulin (Novolin R, Novo Nordisk, Denmark). After the incubation, muscle strips were rinsed for $10 \mathrm{~min}$ at $29^{\circ} \mathrm{C}$ using $2 \mathrm{ml}$ KHB buffer containing $40 \mathrm{mM}$ mannitol and $0.1 \%$ BSA and further incubated for $20 \mathrm{~min}$ at $29^{\circ} \mathrm{C}$ in 1.5 $\mathrm{ml}$ of KHB buffer which contained $8 \mathrm{mM}$ 2-deoxy$\mathrm{D}-\left[1,2-{ }^{3} \mathrm{H}(\mathrm{N})\right]$ glucose $(2.25 \mu \mathrm{Ci} / \mathrm{ml}), 32 \mathrm{mM}\left[{ }^{14} \mathrm{C}\right] \mathrm{man}-$ nitol $(0.3 \mu \mathrm{Ci} / \mathrm{ml}), 2 \mathrm{mM}$ sodium pyruvate, and $0.1 \%$ BSA. Flasks were supplied continuously with $95 \%$ $\mathrm{O}_{2}-5 \% \mathrm{CO}_{2}$ throughout the experiment and insulin was present during the wash and the uptake incubations for measuring insulin-stimulated glucose uptake. The stripes were blotted, weighed, and solubilized with $1 \mathrm{ml} 0.5 \mathrm{~N} \mathrm{NaOH}$. Radioactivity was counted using a liquid scintillation counter. 2-Deoxy$\left[{ }^{3} \mathrm{H}\right] \mathrm{glucose}$ uptake values were corrected for extracellular trapping using $\left[{ }^{14} \mathrm{C}\right]$ mannitol counts. 


\section{Western blot analysis}

Protein contents of $\alpha$ subunit of AMPK (AMPK $\alpha$ ), phosphorylated AMPK $\alpha$ (P-AMPK $\alpha$ ), ACC, phosphorylated ACC (P-ACC) and GluT4 of rats' gastrocnemius muscles were determined using Western blot. Muscles were homogenized in a buffer suitable for preserving phosphorylation states of enzymes, containing $50 \mathrm{mM}$ HEPES, $\mathrm{pH}$ $7.4,150 \mathrm{mM} \mathrm{NaCl}, 10 \%$ glycerol, $1 \%$ Triton X-100, $1.5 \mathrm{mM} \mathrm{MgCl}, 1 \mathrm{mM}$ EDTA, $10 \mathrm{mM} \mathrm{Na} \mathrm{PO}_{4}, 100$ $\mathrm{mM} \mathrm{NaF}, 2 \mathrm{mM} \mathrm{Na} \mathrm{VO}_{4}, 10 \mu \mathrm{g} / \mathrm{ml}$ leupeptin, 10 $\mu \mathrm{g} / \mathrm{ml}$ aprotinin, and $1 \mathrm{mM}$ phenylmethylsulfonyl fluoride, using a Wheaton glass homogenizer and a tight-fitting pestle. Samples for Western blots were prepared from supernatants after centrifugation of homogenates for $10 \mathrm{~min}$ at $14,000 \times \mathrm{g}$ and protein concentrations were determined by Lowry protein assay (Bio-Rad, Hercules, CA). After SDS-PAGE $(10 \%$ resolving gels, except $5 \%$ gels for ACC and P-ACC), proteins were transferred from gel to nitrocellulose membrane. Membranes were blocked in $5 \%$ nonfat dry milk (Sigma) in

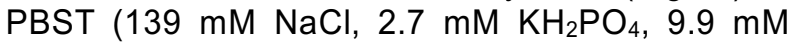
$\mathrm{Na}_{2} \mathrm{HPO}_{4}$, and $0.1 \%$ Tween 20) for $1 \mathrm{~h}$ and then incubated overnight with the specific antibodies to the AMPK $\alpha$ (Cell signaling), P-AMPK $\alpha$ (Cell signaling), ACC (Cell signaling, Berverly, MA), P-ACC (Upstate, Lake Placid, NY) and GluT4 (Santa Cruz, CA). After incubation with the relative second antibody, immune complexes were detected using the ECL method, and immunoreactive bands were quantified using Alphaimager 2200. Values were corrected with the absorbency of the internal control (Actin).

\section{Real-time PCR}

There are two isoforms of AMPK $\alpha: \alpha 1$ and $\alpha 2$. We further determined the mRNA levels of AMPK $\alpha 1$ and AMPK $\alpha 2$ of rats' gastrocnemius muscles using
Real-time PCR. GluT4 mRNA contents were also measured. Total RNA was extracted from frozen gastrocnemius muscle using standard Trizol RNA isolation method. Reverse transcription of $1 \mu \mathrm{g}$ RNA was carried out according to the instructions of TaKaRa RT kit (Invitrogen, Carlsbad, CA). Specific primers designed against rat AMPK $\alpha 1$, AMPK $\alpha 2$, Glut4 and GAPDH were verified in NCBI Blast and primers sequences are described as follows. For AMPK $\alpha$ 1: forward 5'GGGATCCATCAGCAACTATCG3'; reverse 5'GGGAGGTCACGGATGAGG3'. For AMPK $\alpha$ 2: forward 5'CATTTGTGCAAGGCCCCTAGT3'; reverse 5'GACTGTTGGTATCTGCCTGTTTCC3'. For GluT4: forward 5'GGGCTGTGAGTGAGTGCTTTC3'; reverse 5'CAGCGAGGCAAGGCTAGA3'. For GAPDH (internal control): forward 5'TGGTGGACCTCATGGCCTAC3'; reverse 5'CAGCAACTGAGGGCCTCTCT3'.

Real-time PCR was performed using Quantitect SYBR Green kit (Qiagen, Standford, Valencia, CA) following the manufacturer's instructions. Reaction volume was $25 \mu \mathrm{l}$, and $100 \mathrm{ng}$ cDNA were used as the template. The annealing temperature was $57^{\circ} \mathrm{C}$. Fluorescence was detected using ABI Prism 7700 Detection System. PCR products were visualized with gel electrophoresis to confirm a single product of the correct size (150 bp). Ratios of target gene to GAPDH were calculated and compared between samples.

\section{Data analysis}

All experiments were repeated at least three times. Values are given as means \pm S.D. Data were analyzed using SPSS 10.0 software. Statistical significance was assessed by ANOVA and unpaired Student's $t$-test.

Table 1. Rats' body weight, fast plasma glucose levels, serum insulin levels, triglyceride and total cholesterol levels.

\begin{tabular}{ccccccc}
\hline Groups & $n$ & $\begin{array}{c}\text { BW } \\
(\mathrm{g})\end{array}$ & $\begin{array}{c}\mathrm{FG} \\
(\mathrm{mmol} / \mathrm{l})\end{array}$ & $\begin{array}{c}\mathrm{FI} \\
(\mathrm{pmol} / \mathrm{l})\end{array}$ & $\begin{array}{c}\text { Tch } \\
(\mathrm{mmol} / \mathrm{l})\end{array}$ & $\begin{array}{c}\text { TG } \\
(\mathrm{mmol} / \mathrm{l})\end{array}$ \\
\hline Young & 9 & $253 \pm 13$ & $2.83 \pm 0.25$ & $43.6 \pm 2.7$ & $1.67 \pm 0.27$ & $0.86 \pm 0.09$ \\
Old & 9 & $482 \pm 28^{\#}$ & $3.29 \pm 0.15^{\#}$ & $57.8 \pm 4.3^{*}$ & $2.23 \pm 0.1^{*}$ & $1.28 \pm 0.12^{*}$ \\
AICAR & 9 & $478 \pm 17$ & $3.23 \pm 0.22$ & $49 \pm 2.3^{*}$ & $2.31 \pm 0.26$ & $1.31 \pm 0.12$ \\
\hline
\end{tabular}

Rats' bodyweights were measured and biochemical analyses were performed as described in the methods. Compared with young rats (4 month old), old ones (24 month old) exhibited obviously impaired insulin sensitivity, evidenced by elevated body weights $(P<0.01)$, fasting plasma glucose levels $(P<$ $0.01)$ and fasting serum insulin levels $(P<0.05)$. Plasma triglyceride and total cholesterol levels also increased during aging $(P<0.05)$. Compared with old group, AICAR treatment induced a $16 \%$ decrement of fasting insulin levels $(P<0.05)$. No alternations of bodyweights, fasting glucose, plasma triglyceride and total cholesterol levels were detected between old group and AICAR treatment group. Values are given as means $\pm \mathrm{SD}$ and $P<0.05$ is considered statistically significant. BW, body weight; FG, Fasting glucose; FI, Fasting insulin; Tch, total cholesterol; TG, triglyceride; Young, young rats; Old, old rats; AICAR, old rats treated with AICAR. *: $P<0.05 ;{ }^{*}: P<0.01$. 


\section{Results}

\section{Evaluation of insulin sensitivity of aged and AICAR-treated rats}

Rats' body weights, fasting plasma glucose levels, serum insulin levels, triglyceride and total cholesterol levels were measured and results were presented as Table 1. Compared with 4-month aged rats, 24-month old rats exhibited obviously impaired insulin sensitivity, indicated by increased levels of body weight $(P<0.01)$, fasting plasma glucose $(P<0.01)$, fasting serum insulin $(P<$ $0.05)$, as well as plasma triglyceride and total cholesterol levels $(P<0.05)$. Compared with 24month old rats, administration of AICAR induced a $16 \%$ decrease of fasting serum insulin levels $(P<$ $0.05)$, while did not affect rats' body weights, fasting plasma glucose levels and plasma triglyceride and total cholesterol levels.

\section{Aging impairs insulin-induced glucose uptake of rats' muscles}

To further determine insulin sensitivity of rats' skeletal muscle, in vitro glucose uptake of isolated gastrocnemius muscles were measured. Data were provided as Figure 1. Compared with 4-month aged rats, both basal and insulin-stimulated glucose uptake significantly decreased in 24-month old rats $(P<0.05)$, indicating onset of insulin resistance. Compared with 24-month aged rats, AICAR

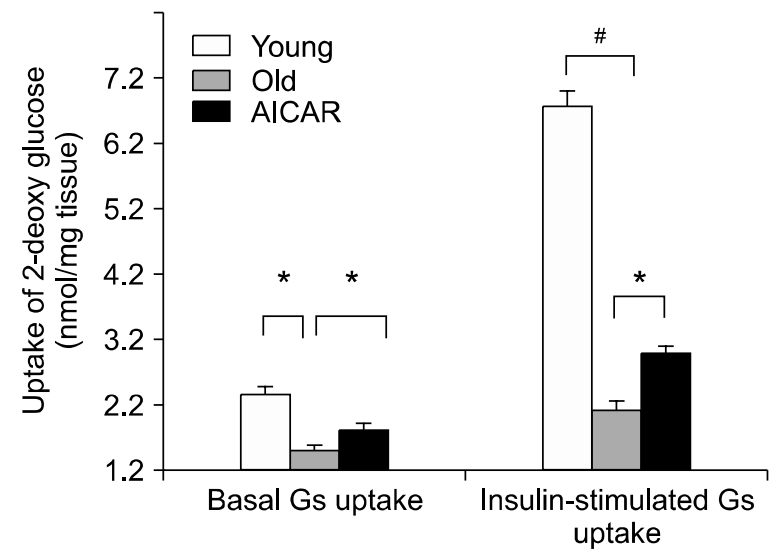

Figure 1. Aging impairs insulin-induced glucose uptake of rats' muscles. Compared with young rats, aging induced a $37 \%$ decrease of basal glucose uptake $(P<0.05)$ and a $69 \%$ decrease in insulin-stimulated glucose uptake $(P<0.01)$. When compared with old rats, administration of AICAR increase basal glucose uptake by 1.2 fold and insulin-stimulated glucose uptake by 1.4 fold $(P<0.05)$. Values are given as means \pm SD and $P<0.05$ is considered statistically significant. Young, young rats; Old, old rats; AICAR, old rats treated with AICAR. *: $P<0.05$; *: $P$ $<0.01$.

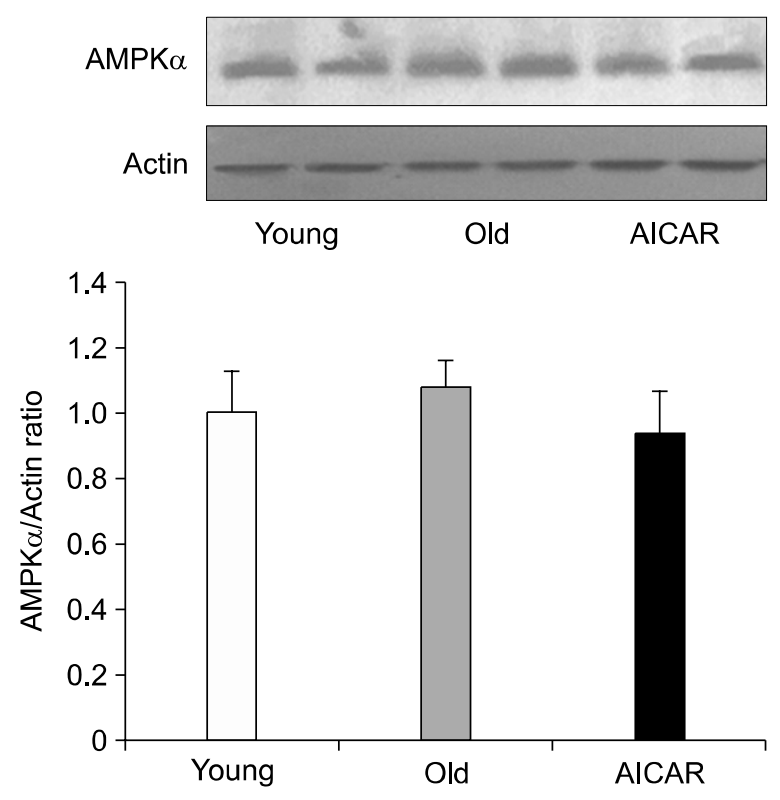

Figure 2. Aging does not affect AMPK $\alpha$ protein levels. AMPK $\alpha$ protein contents of rats' gastrocnemius muscle were quantified using western blot. No significant alternations were detected among three groups. Values are given as means $\pm S D$ and $P<0.05$ is considered statistically significant. Young, young rats; Old, old rats; AICAR, old rats treated with AICAR.

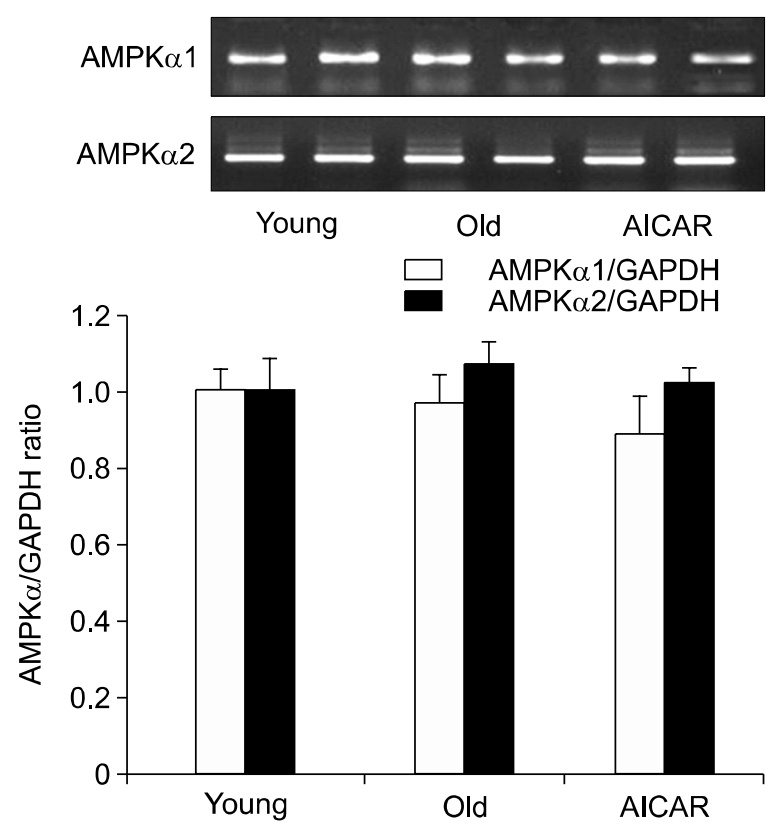

Figure 3. mRNA levels of AMPK $\alpha 1$ and AMPK $\alpha 2$ does not alter during aging. mRNA levels of AMPK $\alpha 1$ and AMPK $\alpha 2$ in rats' gastrocnemius muscle were determined using Real-time PCR. No alternations of AMPK $\alpha 1$ and AMPK $\alpha 2$ mRNA expression were detected among three groups. Values are given as means $\pm \mathrm{SD}$ and $P<0.05$ is considered statistically significant. Young, young rats; Old, old rats; AICAR, old rats treated with AICAR. 
treatment induced a 1.2 fold increase of basal glucose uptake $(P<0.05)$, and 1.4 fold increase of insulin-stimulated glucose uptake $(P<0.05)$.

\section{Expression of AMPK $\alpha$ does alter during aging}

To determine the effects of aging on the expression of AMPK $\alpha$, protein contents of total AMPK $\alpha$ and mRNA levels of AMPK $\alpha 1$ and AMPK $\alpha 2$ of rats' gastrocnemius muscle were quantified using Western blot and Real-time PCR separately. No significant differences of AMPK $\alpha$ protein levels were detected between 4-month old rats and with 24-month old rats (Figure 2). Consistent with such findings, mRNA levels of AMPK $\alpha 1$ and AMPK $\alpha 2$ also did not change during aging (Figure 3), indicating aging might not affect AMPK $\alpha$ expression. Compared with 24-month old rats, no alternations of AMPK- $\alpha$ protein and mRNA levels were detected after AICAR treatment (Figure 2 and 3).

\section{Aging impairs AMPK $\alpha$ activities in rat' muscles}

Activities of AMPK $\alpha$ were evaluated by measuring the protein contents of P-AMPK $\alpha$, its active state, in rats' gastrocnemius muscles. As shown in Figure 4, 24-month aged rats exhibited significant

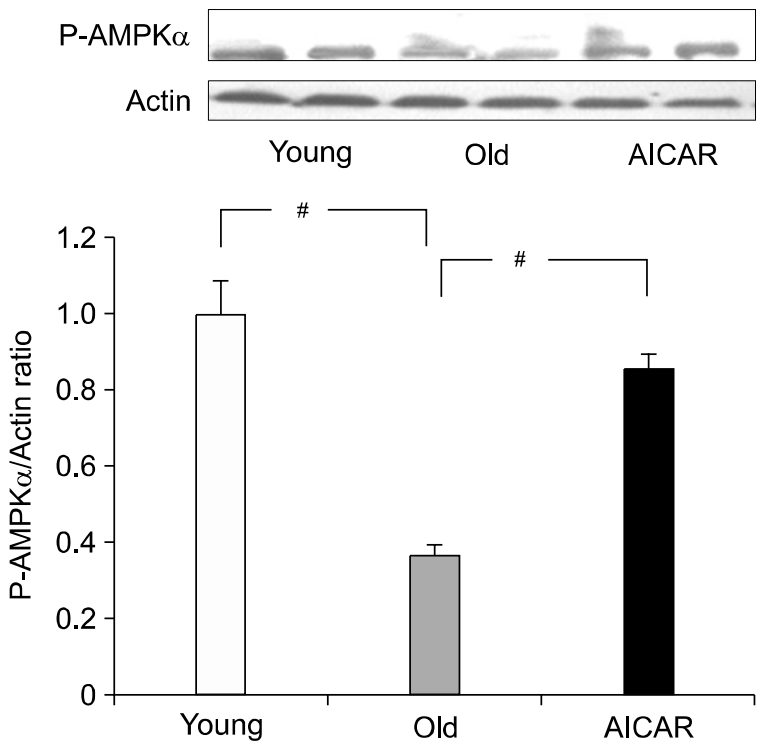

Figure 4. Aging impairs AMPK $\alpha$ activities. Protein levels of P-AMPK $\alpha$ in rats' gastrocnemius muscle were measured to evaluate AMPK $\alpha$ activities. Compared with young rats, P-AMPK $\alpha$ protein levels decreased by $63 \%$ in old rats $(P<0.01)$, suggesting aging might impair AMPK $\alpha$ activities. Compared with old rats, AICAR induced a $132 \%$ increment of P-AMPK $\alpha$ protein levels $(P<0.01)$. Values are given as means \pm SD and $P<0.05$ is considered statistically significant. Young, young rats; Old, old rats; AICAR, old rats treated with AICAR. ${ }^{\#}: P<0.01$. decreased P-AMPK $\alpha$ protein contents compared with that of 4-month old $(P<0.01)$, suggesting aging might impair AMPK $\alpha$ activities. AICAR is a known activator of AMPK, and its AMPK-activating effect was also demonstrated in the present study. Compared with 24-month old rats, AICAR treatment induced a $132 \%$ increment of P-AMPK $\alpha$ levels $(P<0.01)$.

\section{P-ACC levels decreased during aging}

The major biological effects of AMPK $\alpha$ are to phosphorylate and inactivate ACC. To further investigate the effects of aging on AMPK $\alpha$, protein levels of P-ACC and ACC of rats' gastrocnemius muscles were determined using specific antibodies (Figure 5). No alternations of ACC levels were detected among three groups. However, aging induced a $51 \%$ decrease of P-ACC protein contents $(P<0.05)$. Compared with 24-month old rats, administration of AICAR elevated P-ACC levels by $94 \%(P<0.05)$. These findings not only

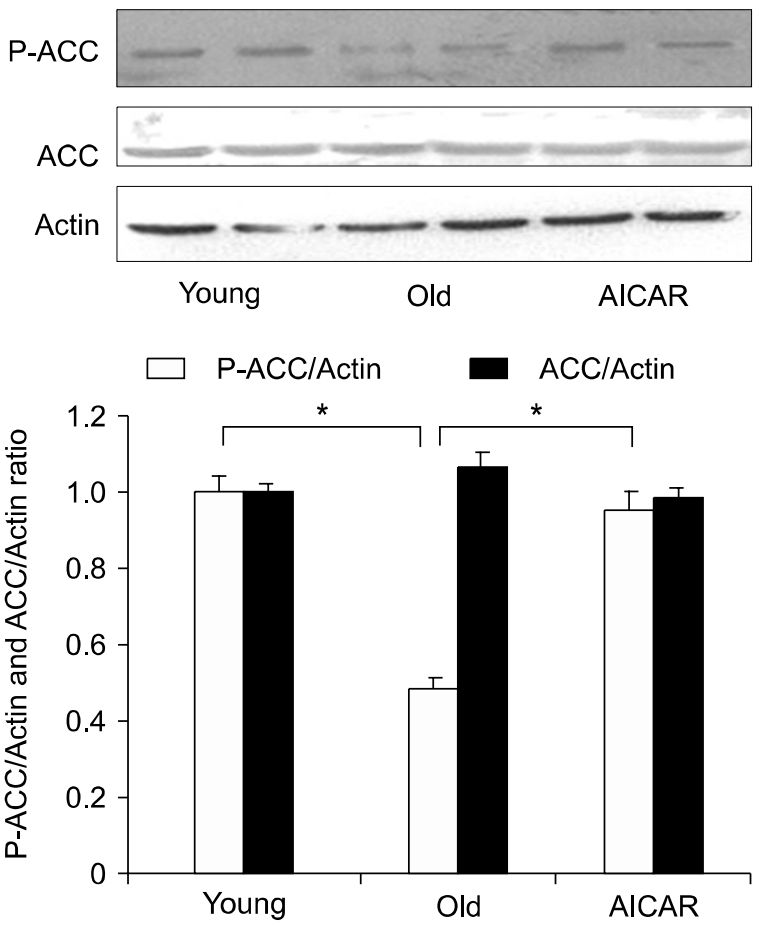

Figure 5. P-ACC levels decreased during aging. Protein contents of ACC and P-ACC in rats' gastrocnemius muscle were determined using Western blot. Compared with young rats, 24-month aged rats exhibited $51 \%$ decrement of P-ACC protein contents $(P<0.05)$. When compared with old rats, administration of AICAR increased P-ACC protein levels by $94 \%(P<0.05)$. No alternations of ACC protein levels were detected among three groups. Values are given as means $\pm \mathrm{SD}$ and $P$ $<0.05$ is considered statistically significant. Young, young rats; Old, old rats; AICAR, old rats treated with AICAR. *: $P<0.05$. 
present further evidences suggesting aging might impair AMPK $\alpha$ activities, but also indicate the ACC-mediated pathway might act as the downstream player underlying aging-related fatty acid oxidation insufficiency and insulin resistance.

\section{Aging decreased GluT4 expression}

Besides regulating intracellular fat oxidation, AMPK has also been evidenced it could regulate insulin sensitivity via promoting GluT4 expression (Jessen et al., 2003). Aging-induced insulin resistance is accompanied with decreased GluT4 expression (Houmard et al., 1995; Larkin et al., 2001), the same effect was also demonstrated in the present study (Figure 6). 24-month old rats showed a 71\% decrease of GluT4 protein levels and 53\% decrement of GluT4 mRNA levels, compared with 4-month aged rats $(P<0.05)$. The impairment of

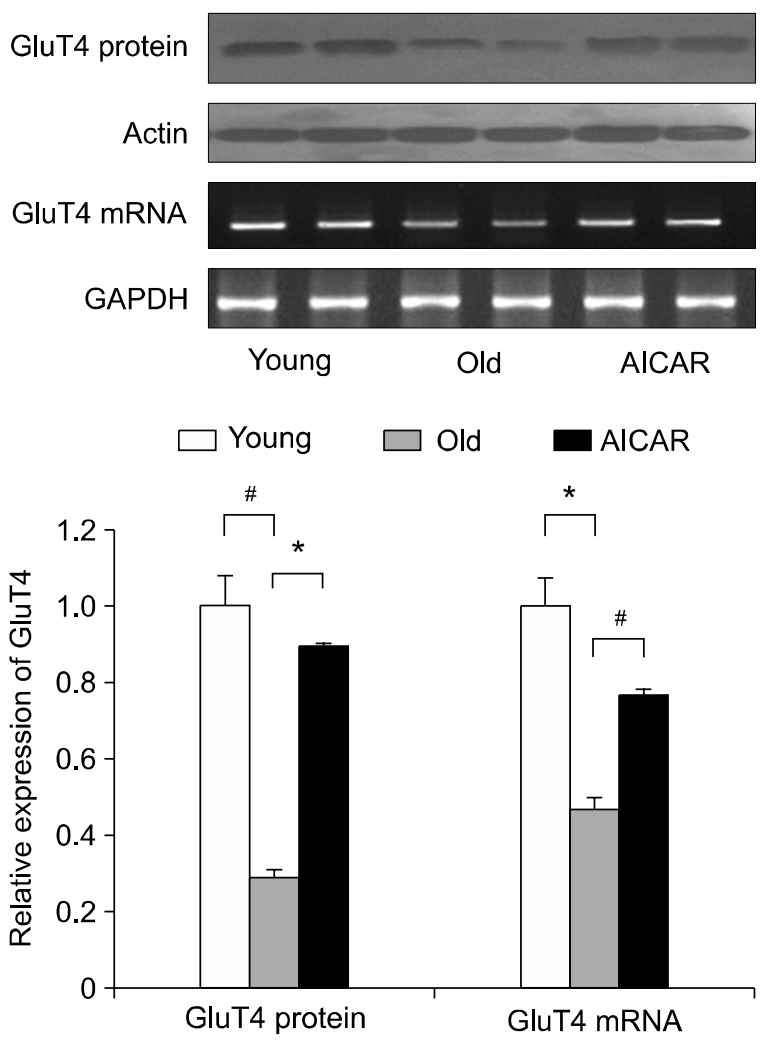

Figure 6. Aging decreased GluT4 expression. Protein and mRNA levels of GluT4 in rats' gastrocnemius muscle were measured using Western blot and Real-time PCR. Compared with young rats, aging induced a $71 \%$ decrease of GluT4 protein levels and a $53 \%$ decrease of its $\mathrm{mRNA}$ levels $(P<0.05)$. Both the protein and mRNA levels of GluT4 significantly elevated after AICAR treatment $(P<0.05)$. Values are given as means $\pm S D$ and $P<0.05$ is considered statistically significant. Young, young rats; Old, old rats; AICAR, old rats treated with AICAR. * $P$ $<0.05$;: $P<0.01$.
GluT4 expression during aging was significantly ameliorated by AICAR treatment $(P<0.05)$. These data indicate GluT4 might act as another downstream player of AMPK $\alpha$ in the pathogenesis of aging-related insulin resistance.

\section{Discussion}

As proposed by professor McGarry JD (McGarry, 2002), the inefficient intracellular fatty acid oxidation and accordingly increased intracellular fat accumulation in insulin target organs are important contributors and predictors of insulin resistance. To date, the relationship between intracellular fatty acid metabolism and insulin sensitivity has been well documented by both in-vivo and in-vitro studies. For example, it was showed animals treated with R-etomoxir, an inhibitor of CPT-1, the key enzyme of intracellular fatty acid beta-oxidation, became more insulin resistant (Dobbins et al., 2001), while those with CPT-1 over-expression exhibited significantly increased insulin sensitivity (Perdomo et al., 2004). Aging is also associated with impaired intracellular fatty acid oxidation (Calles-Escandon et al., 1995, 1997; Park et al., 2006), and this was proposed as an important mechanism underlying age-related insulin resistance (Petersen et al., 2003; Cree et al., 2004; lossa et al., 2004; Menshikova et al., 2006; Slawik and Vidal-Puig, 2006). However, the mechanisms are still unclear. As described above, neither altered body composition nor insufficient physical exercise could fully explain such the defects. Thus, the precise mechanism underlying still needs further investigation.

AMPK, via regulating ACC activities and intracellular malonyl-CoA contents, has been evidenced as an important regulator of intracellular fat acid oxidation and insulin sensitivity (Rutter et al., 2003). Furthermore, it was demonstrated recently that altered expression or activity of AMPK might play a key role in the onset of both high-fat and high-glucose induced insulin resistance (Kraegen et al., 2006; Liu et al., 2006). In the present study, we demonstrated AMPK might also participate in aging-related fat oxidation insufficiency and insulin resistance. Both the expression and activity of AMPK $\alpha$ were investigated using Western blot and Real-Time PCR separately. Aging might not affect AMPK $\alpha$ expression, as neither mRNA levels of AMPK $\alpha 1$ and AMPK $\alpha 2$ nor protein contents of total AMPK $\alpha$ altered between 4-month aged and 24-month aged rats. However, protein levels of P-AMPK $\alpha$, the active form of AMPK $\alpha$, significantly decreased during aging, suggesting impaired 
AMPK $\alpha$ activities. Thus, our findings present direct evidences that aging-related insulin resistance is also associated with decreased AMPK $\alpha$ activity.

As mentioned above (Rutter et al., 2003), the major biological effect of AMPK is to regulate intracellular fatty acid oxidation via inactivating ACC in a phosphorylation manner. We further investigated the activity of ACC. Although no alternations of total ACC levels were detected between 4-month aged and 24-aged rats, P-ACC contents significantly decreased during aging. ACC is the key regulator of intracellular malonyl-CoA production and the phosphorylation state is its inactive form. The reduced P-ACC levels would unavoidably lead to the enhanced ACC activity, increased malonyl-CoA contents, impaired intracellular fatty acid oxidation and accordingly, insulin resistance. Thus, ACC might act as a downstream factor in the possible AMPK-mediated mechanism underlying aging-related intracellular fat oxidation insufficiency and insulin resistance. Enhanced ACC activities could lead to onset of insulin resistance, while inactivating ACC has insulin-sensitizing effects, as demonstrated by Abu-Elheiga $\mathrm{L}$ and his colleagues (Abu-Elheiga et al., 2003). In their studies, ACC-deactivated animals, which were created either by gene knockout or gene mutant, exhibited obviously enhanced intracellular fatty acid oxidation and were protected from high-fat induced resistance. The alternations of $P$-ACC levels during aging might not only provide further evidences to our suggestions that AMPK $\alpha$ plays a role in aging-associated inefficient intracellular fat oxidation and insulin resistance, but also suggest a possible ACC-mediated pathway might be involved.

Besides regulating insulin sensitivity via affecting ACC activities and intracellular fat acid oxidation as described above, AMPK could directly regulate GluT4 expression (Jessen et al., 2003; Holmes et al., 2005). In cultured skeletal muscle cells, overexpression of an active mutant AMPK $\alpha$ subunit induced a significant increment of GluT4 protein contents, along with increased insulin-stimulated glucose uptake (Fryer et al., 2002). The same effects have also been demonstrated by in-vitro studies. As demonstrated by Jessen et al. (2003), a single injection of AICAR resulted in an almost 2 fold increment of Glut4 protein contents in rats' skeletal muscles. Aging-induced insulin resistance is also associated with decreased GluT4 expression, which has been well documented in both humans (Houmard et al., 1995) and rodents (Larkin et al., 2001), as well as in our present research. Although other mechanisms could not be excluded, impaired AMPK $\alpha$ activities might contri- bute, at least partly, to age-related decrease of GluT4 expression. Thus, Glut4 might act as another downstream effecter in the AMPK-mediated mechanism underlying aging-induced insulin resistance, besides regulating fatty acid oxidation via ACC. MEF2 and GEF are two transcription regulators of Glut4, and AMPK has been demonstrated recently it could regulate their expressions and activities (Holmes et al., 2005). Whether such the mechanism is also involved in aging-induced insulin resistance needs further investigation.

AICAR is the most widely used activator of AMPK. To further determine the role of AMPK $\alpha$ in age-related insulin resistance, AICAR was also employed in our present study and its AMPKactivating effect was also confirmed by measuring the P-AMPK $\alpha$ and P-ACC levels. Accompanied with activation of AMPK, rats treated with AICAR exhibited obviously ameliorated aging-related glucose metabolism disturbance, indicated by increased Glut4 contents, enhanced insulinstimulated glucose uptake, and decreased fasting serum insulin levels. Thus, these data put further evidences supporting the role of AMPK in aginginduced insulin resistance.

In conclusion, our present research suggested aging is associated with impaired AMPK $\alpha$ activity and such the impairment might contribute, at least partly, to aging-related insulin resistance. Similar to our suggestions, it was demonstrated recently AMPK plays a fundamental role in several signal pathways controlling aging process (Curtis et al., 2006). Also, it was proposed and subsequently was evidenced that activating AMPK has the effect of slowing aging and extending lifespan (McCarty, 2004; Curtis et al., 2006). Although the relationship between aging and AMPK has been well documented, little is known about the upstream mechanisms responsible for aging-related reductions of AMPK. Cellular AMPIATP ratio, LKB1 and Calmodulin-dependent protein kinase kinase $\beta$ (CaMKK $\beta$ ) are all physiological regulators of AMPK. Whether aging-related decrement of AMPK activity is also mediated by these regulators needs further investigation.

\section{Acknowledgments}

This research was supported by Shandong Doctoral Foundation (2006BSB14022).

\section{References}

Abu-Elheiga L, Oh W, Kordari P, Wakil SJ. Acetyl-CoA carboxylase 2 mutant mice are protected against obesity and 
diabetes induced by high-fat/high-carbohydrate diets. Proc Natl Acad Sci USA 2003;100:10207-12

Boirie Y, Gachon P, Cordat N, Ritz P, Beaufrere B. Differential insulin sensitivities of glucose, amino acid, and albumin metabolism in elderly men and women. J Clin Endocrinol Metab 2001;86:638-44

Calles-Escandon J, Arciero PJ, Gardner AW, Bauman C, Poehlman ET. Basal fat oxidation decreases with aging in women. J Appl Physiol 1995;78:266-71

Calles-Escandon J, Poehlman ET, Garcia-Rubi E. Lipolysis in elderly postmenopausal women. Metabolism 1997; 46:1312-5

Carvalho CR, Brenelli SL, Silva AC, Nunes AL, Velloso LA, Saad MJ. Effect of aging on insulin receptor, insulin receptor substrate-1, and phosphatidylinositol 3-kinase in liver and muscle of rats. Endocrinology 1996;137:151-9

Cox JH, Cortright RN, Dohm GL, Houmard JA. Effect of aging on response to exercise training in humans: skeletal muscle GLUT-4 and insulin sensitivity. J Appl Physiol 1999;86: 2019-25

Cree MG, Newcomer BR, Katsanos CS, Sheffield-Moore M, Chinkes D, Aarsland A, Urban R, Wolfe RR. Intramuscular and liver triglycerides are increased in the elderly. J Clin Endocrinol Metab 2004;89:3864-71

Curtis R, O'Connor G, DiStefano PS. Aging networks in Caenorhabditis elegans: AMP-activated protein kinase (aak-2) links multiple aging and metabolism pathways. Aging Cell 2006;5:119-26.

Dobbins RL, Szczepaniak LS, Bentley B, Esser V, Myhill J, McGarry JD. Prolonged inhibition of muscle carnitine palmitoyltransferase-1 promotes intramyocellular lipid accumulation and insulin resistance in rats. Diabetes 2001; 52:123-30

Fryer LG, Foufelle F, Barnes K, Baldwin SA, Woods A, Carling D. Characterization of the role of the AMP-activated protein kinase in the stimulation of glucose transport in skeletal muscle cells. Biochem J 2002;363:167-74

Holmes BF, Sparling DP, Olson AL, Winder WW, Dohm GL. Regulation of muscle GLUT4 enhancer factor and myocyte enhancer factor 2 by AMP-activated protein kinase. Am J Physiol Endocrinol Metab 2005;289:E1071-6

Houmard JA, Weidner MD, Dolan PL, Leggett-Frazier N, Gavigan KE, Hickey MS, Tyndall GL, Zheng D, Alshami A, Dohm GL. Skeletal muscle GLUT4 protein concentration and aging in humans. Diabetes 1995;44:555-60

lossa S, Mollica MP, Lionetti L, Crescenzo R, Tasso R, Liverini G. A possible link between skeletal muscle mitochondrial efficiency and age-induced insulin resistance. Diabetes 2004;53:2861-6.

Jessen N, Pold R, Buhl ES, Jensen LS, Schmitz O, Lund S. Effects of AICAR and exercise on insulin-stimulated glucose uptake, signaling, and GLUT-4 content in rat muscles. J Appl Physiol 2003;94:1373-9

Kraegen EW, Saha AK, Preston E, Wilks D, Hoy AJ, Cooney GJ, Ruderman NB. Increased malonyl-CoA and diacylglycerol content and reduced AMPK activity accompany insulin resistance induced by glucose infusion in muscle and liver of rats. Am J Physiol Endocrinol Metab 2006;290: E471-9

Larkin LM, Reynolds TH, Supiano MA, Kahn BB, Halter JB. Effect of aging and obesity on insulin responsiveness and glut-4 glucose transporter content in skeletal muscle of Fischer 344 x Brown Norway rats. J Gerontol A Biol Sci Med Sci 2001;56:B486-92

Liu Y, Wan Q, Guan Q, Gao L, Zhao J. High-fat diet feeding impairs both the expression and activity of AMPKa in rats' skeletal muscle. Biochem Biophys Res Commun 2006; 339:701-7

McCarty MF. Chronic activation of AMP-activated kinase as a strategy for slowing aging. Med Hypotheses 2004;63: 334-9

McGarry JD. Banting lecture 2001: dysregulation of fatty acid metabolism in the etiology of type 2 diabetes. Diabetes 2002; 51:7-18

Menshikova EV, Ritov VB, Fairfull L, Ferrell RE, Kelley DE, Goodpaster BH. Effects of exercise on mitochondrial content and function in aging human skeletal muscle. J Gerontol A Biol Sci Med Sci 2006;61:534-40

Minokoshi Y, Kim YB, Peroni OD, Fryer LG, Muller C, Carling D, Kahn BB. Leptin stimulates fatty-acid oxidation by activating AMP-activated protein kinase. Nature 2002; 415:339-43

Park SY, Kim YW, Kim JE, Kim JY. Age-associated changes in fat metabolism in the rat and its relation to sympathetic activity. Life Sci 2006;79:2228-33

Perdomo G, Commerford SR, Richard AM, Adams SH, Corkey BE, O'Doherty RM, Brown NF. Increased betaoxidation in muscle cells enhances insulin-stimulated glucose metabolism and protects against fatty acid-induced insulin resistance despite intramyocellular lipid accumulation. J Biol Chem 2004;279:27177-86

Petersen KF, Befroy D, Dufour S, Dziura J, Ariyan C, Rothman DL, DiPietro L, Cline GW, Shulman GI. Mitochondrial dysfunction in the elderly: possible role in insulin resistance. Science 2003;300:1140-2

Rutter GA, Da Silva Xavier G, Leclerc I. Roles of 5'-AMPactivated protein kinase (AMPK) in mammalian glucose homoeostasis. Biochem J 2003;375:1-16

Scheen AJ. Diabetes mellitus in the elderly: insulin resistance and/or impaired insulin secretion? Diabetes Metab 2005;31:5S27-34

Seals DR, Hagberg JM, Hurley BR, Ehsani AA, Holloszy JO. Effects of endurance training on glucose tolerance and plasma lipids levels in old men and women. JAMA 1984; 252:645-9

Short KR, Vittone JL, Bigelow ML, Proctor DN, Rizza RA, Coenen-Schimke JM, Nair KS. Impact of aerobic exercise training on age-related changes in insulin sensitivity and muscle oxidative capacity. Diabetes 2003;52:1888-96

Sial S, Coggan AR, Carroll R, Goodwin J, Klein S. Fat and carbohydrate metabolism during exercise in elderly and young subjects. Am J Physiol Endocrinol Metab 1996; 
271:E983-9

Sial S, Coggan AR, Hickner RC, Klein S. Training-induced alterations in fat and carbohydrate metabolism during exercise in elderly subjects. Am J Physiol Endocrinol Metab 1998;274:E785-90

Slawik M, Vidal-Puig AJ. Lipotoxicity, overnutrition and energy metabolism in aging. Ageing Res Rev 2006;5:144-64

Wan Q, Liu Y, Guan Q, Gao L, Lee KO, Zhao J. Ethanol feeding impairs insulin-stimulated glucose uptake in isolated rat skeletal muscle: role of Gs alpha and cAMP. Alcohol Clin Exp Res 2005;29:1450-6
Yamauchi T, Kamon J, Minokoshi Y, Ito Y, Waki H, Uchida S, Yamashita S, Noda M, Kita S, Ueki K, Eto K, Akanuma Y, Froguel P, Foufelle F, Ferre P, Carling D, Kimura S, Nagai R, Kahn BB, Kadowaki T. Adiponectin stimulates glucose utilization and fatty-acid oxidation by activating AMP-activated protein kinase. Nat Med 2002;8:1288-95

Zhou G, Myers R, Li Y, Chen Y, Shen X, Fenyk-Melody J, Wu M, Ventre J, Doebber T, Fujii N, Musi N, Hirshman MF, Goodyear LJ, Moller DE. Role of AMP-activated protein kinase in mechanism of metformin action. J Clin Invest 2001;108:1167-74 